\title{
Label-free graphene biosensor targeting cancer molecules based on non- covalent modification
}

Zhou, L. ; Mao, H.; Wu, C. ; Tang, L.; Wu, Z.; Sun, H.; Zhang, H.; Zhou, H.; Jia, C.; Jin, Q.; Chen, Xianfeng; Zhao, J.

\section{Biosensors and Bioelectronics}

DOI:

10.1016/j.bios.2016.09.025

Published: 15/01/2018

Peer reviewed version

Cyswllt i'r cyhoeddiad / Link to publication

Dyfyniad o'r fersiwn a gyhoeddwyd / Citation for published version (APA):

Zhou, L., Mao, H., Wu, C., Tang, L., Wu, Z., Sun, H., Zhang, H., Zhou, H., Jia, C., Jin, Q., Chen, X., \& Zhao, J. (2018). Label-free graphene biosensor targeting cancer molecules based on noncovalent modification. Biosensors and Bioelectronics, 87, 701-707.

https://doi.org/10.1016/j.bios.2016.09.025

\footnotetext{
Hawliau Cyffredinol / General rights

Copyright and moral rights for the publications made accessible in the public portal are retained by the authors and/or other copyright owners and it is a condition of accessing publications that users recognise and abide by the legal requirements associated with these rights.

- Users may download and print one copy of any publication from the public portal for the purpose of private study or research.

- You may not further distribute the material or use it for any profit-making activity or commercial gain

- You may freely distribute the URL identifying the publication in the public portal ?
}

Take down policy

If you believe that this document breaches copyright please contact us providing details, and we will remove access to the work immediately and investigate your claim. 


\section{Label-free graphene biosensor targeting cancer molecules based on non-covalent modification}

Lin Zhou ${ }^{1,2}$, Hongju Mao ${ }^{1 *}$,Chunyan $\mathrm{Wu}^{1,3}$, Lin Tang ${ }^{1,4}$, Zhenhua $\mathrm{Wu}^{1,2}$, Hao Sun ${ }^{1}$, Honglian Zhang ${ }^{1}$, Hongbo Zhou $^{1}$,Chunping Jia ${ }^{1}$, Qinghui Jin ${ }^{1}$, Xianfeng Chen ${ }^{5}$, Jianlong Zhao $^{1 *}$

${ }^{1}$ State Key Laboratory of Transducer Technology; Key Laboratory of Terahertz Solid-State Technology, Shanghai Institute of Microsystem and Information Technology, Chinese Academy of Sciences, Shanghai 200050, China.

${ }^{2}$ University of Chinese Academy of Sciences, Beijing 100039, China.

${ }^{3}$ Shanghai University, Shanghai, 200031, China.

${ }^{4}$ ShanghaiTech University, Shanghai, 200444, China.

${ }^{5}$ School of Electronic Engineering, Bangor University, Bangor LL57 1UT, United Kingdom.

*Corresponding author. Tel: +86-21-62511070-8705; Fax: +86-21-62511070-8714;

E-mail: hjmao@mail.sim.ac.cn.

*Corresponding author. Tel: +86-21-62511070-8702; Fax: +86-21-62511070-8714;

E-mail: jlzhao@mail.sim.ac.cn. 


\begin{abstract}
A label-free immunosensor based on antibody-modified graphene field effect transistor (GFET) was presented. Antibodies targeting carcinoembryonic antigen (Anti-CEA) were immobilized to the graphene surface via non-covalent modification. The bifunctional molecule, 1-pyrenebutanoic acid succinimidyl ester, which is composed of a pyrene and a reactive succinimide ester group, interacts with graphene non-covalently via $\pi$-stacking. The succinimide ester group reacts with the amine group to initiate antibody surface immobilization, which was confirmed by X-ray Photoelectron Spectroscopy, Atomic Force Microscopy and Electrochemical Impedance Spectroscopy. The resulting anti-CEA modified GFET sufficiently monitored the reaction between CEA protein and anti-CEA in real-time with high specificity, which revealed selective electrical detection of CEA with a limit of detection (LOD) of less than $100 \mathrm{pg} / \mathrm{ml}$. The dissociation constant between CEA protein and anti-CEA was estimated to be $6.35 \times 10^{-11} \mathrm{M}$, indicating the high affinity and sensitivity of anti-CEA-GFET. Taken together, the graphene biosensors provide an effective tool for clinical application and point-of-care medical diagnostics.
\end{abstract}

Keywords: Graphene; Non-covalent modification; CEA protein; Dissociation constant 


\section{Introduction}

Cancer has become one of the most threatening diseases all over the world. Cancer biomarkers are critical in cancer diagnosis and real-time monitoring of health status for patients (Biomarkers Definitions Working 2001). Multiple protein biomarkers are widely distributed in serum, tissues and saliva, and are effective indicators of disease state. In many cases, the detection of these abnormal markers at early stages of disease allows for rapid initiation of appropriate treatments, thereby largely increasing the overall chances of survival for cancer patients. As such, detection of cancer biomarkers is critical in diagnosis, prognosis, and prediction of treatment efficacy and recurrence of cancer

\section{(Giljohann and Mirkin 2009).}

Traditional biomarker detection methods include enzyme linked immunosorbent assay (Voller et al. 1978), radioimmunoassay (Goldsmith 1975), multiplex protein assay (Kingsmore 2006), lateral flow immunoassay (Posthuma-Trumpie et al. 2009), fluorescent microarray and electrochemical methods (Chikkaveeraiah et al. 2012; Zajac et al. 2007). In these methods, expensive reagents and complex processes of data acquisition and quantification are required to achieve high sensitive detection. More importantly, most of the reactions cannot be monitored quantitatively in real-time. So a simple, inexpensive and highly specific detection method would allow for the assessment of target molecules in real-time, which would have broad clinical applications.

Graphene is a single-atom-thick, $\mathrm{sp}^{2}$-hybridized carbon-based material. Due to its planar geometry and good electrical property (Geim and Novoselov 2007; Novoselov et al. 2004), graphene is an ideal candidate for biochemical sensors. The electrical detection of various molecules using graphene and graphene-like materials has been used in a number of reported devices (Schedin et al. 2007). Graphene field-effect transistors (GFETs) have been developed to detect DNA hybridization (Xu et al. 2014), 
glucose (Huang et al. 2010), absorbed proteins and immunoglobulin E (Ohno et al. 2010; Ohno et al. 2009).

Modification of the basal plane of graphene offers a way to control its structure and electronic properties. Covalent binding approaches disrupt the electronic structure of graphene and take place preferentially at carbon atoms near defects and grain boundaries (Sun et al. 2010). Non-covalent functionalization strategies offer the possibility of binding functional groups to graphene without disturbing the electronic network (Chen et al. 2001; Georgakilas et al. 2012; Kodali et al. 2011). Pyrene derivatives have been found to interact non-covalently with graphene via $\Pi$-stacking (Parviz et al. 2012). Among these derivatives, 1-pyrenebutanoic acid succinimidyl ester (PYR-NHS) contains aromatic pyrenyl group to interact with graphene non-covalently and succinimidyl ester group to bind amino probes, which can be used as a non-covalent linker to immobilize proteins such as glucose oxidase and glutamic dehydrogenase (Huang et al. 2010) as well as aptamers on carbon nanotube or graphene surfaces (Chen et al. 2001; Kodali et al. 2011; Ohno et al. 2010).

Two important factors are considered in the use of GFETs as biochemical sensors. The first one is Debye screening length, which is defined as the typical distance, required for screening the charge of target molecules and macromolecules by the dissolved counter ions in a solution. For aqueous solutions at room temperature, Debye screening length depends on the ionic strength and mobile charges on the channel surface of GFET (Stern et al. 2007). The interaction between the capture probes and target molecules must occur within one Debye length. Therefore, the size of capture probes must correlate with the ionic strength of the accompanying buffer solution. Another consideration is the modification of the specificity of the capture probes without disrupting the single layer of graphene in order to optimize the electric properties of graphene. 
Here, this study demonstrates a label-free antibody-modified graphene immunosensor based on noncovalent modification. The target protein is carcinoembryonic antigen (CEA), which can be used to assess prognosis and monitor the progress of cancer (Casey and Kofinas 2008; Haggarty et al. 1986). The aromatic pyrenyl group of PYR-NHS interacts strongly with the plane of graphite via $\Pi$-stacking (Jaegfeldt et al. 1983; Katz 1994). The succinimidyl ester groups are highly reactive to nucleophilic substitution by primary and secondary amines that exist in abundance on the surface of the anticarcinoembryonic antigen (anti-CEA), resulting in the immobilization of anti-CEAs antibodies on the single layer graphene without disrupting its electronic network. Anti-CEA-modified GFET can detect CEA with high specificity and a LOD of less than $100 \mathrm{pg} / \mathrm{ml}$. The dissociation constant between CEA protein and anti-CEA was estimated to be approximately $6.35 \times 10^{-11} \mathrm{M}$ by hill model. Taken together, this technology will have broad applications in the clinical detection of cancer.

\section{Material and methods}

\subsection{Materials and reagents}

Monoclonal antibody anti-carcinoembryonic antigen (anti-CEA) and carcinoembryonic antigen (CEA) protein were purchased from Medix Biochemica (Joensuu, Finland). Neuron specific enolase (NSE) was obtained from Fitzerald (Acton, USA) and Cytokerantin-19-fragment (CYFRA21-1) was purchased from Calbioreagents (California, USA). Polydimethylsiloxane (PDMS) were used to fabricate reactive chamber.1-pyrenebutanoic acid succinimidyl ester (PYR-NHS) and ethanolamine were obtained from Anaspec (California, USA) and Sigma-Aldrich (St. Louis, MO), respectively. Large-sized graphene films on $\mathrm{Cu}$ film were bought from 2D Carbon Tech Inc. (Changzhou, China). Ultra-pure water, obtained from Millipore Q purification system (Millipore, USA), was used for the preparation of all solutions. 


\subsection{Device fabrication}

Titanium/gold metal electrodes were fabricated by photolithography, metal deposition, and lift-off process on the substrate. The single layer graphene on $\mathrm{Cu}$ film generated by chemical vapor deposition was prepared for sensing material, by covering with a polymethyl methacrylate (PMMA) film and subsequently etching the graphene film using an ammonium persulfate aqueous solution, followed by a 5 min rinse in deionized water for three times and the PMMA/graphene film was then placed onto metal electrodes deposited on the substrate. The PMMA/graphene film on the substrate dried in air for $30 \mathrm{~min}$ at room temperature and baked at $80^{\circ} \mathrm{C}$ for $30 \mathrm{~min}$. The substrate was then immersed in acetone to dissolve the PMMA, leaving the graphene films still attached on the metal electrodes for subsequent device fabrication. The channel of GFETs was defined by photolithography, and the surrounding graphene was etched by plasma. Finally, the surface of the graphene channel was cleaned by annealing in pure $\mathrm{Ar}$ atmosphere at $400^{\circ} \mathrm{C}$, and then the reaction chamber was anchored on the substrate using silicone. The reaction chamber was made of PDMS. One terminal of the miniaturized $\mathrm{Ag} / \mathrm{AgCl}$ electrochemical reference electrode was fixed on the shelf and another terminal of electrochemical reference electrode was immersed in the reactive chamber as the gate.

\subsection{Modification of GFET}

To achieve non-covalent modification of the graphene surface, GFET was incubated in dry dimethylformamide (DMF) solution of 5mM PYR-NHS, which served as a linker, for $2 \mathrm{~h}$ at room temperature, followed by rinsing with DMF. In order to covalently immobilize anti-CEA on the graphene surface, the device was exposed to $2 \mathrm{mg} / \mathrm{mL}$ anti-CEA antibody in $10 \mathrm{mM}$ phosphate buffer solution (pH 7.2) overnight. After immobilizing anti-CEA and rinsing with blank PBS, $100 \mathrm{mM}$ ethanolamine was added onto the channel of the GFET for $1 \mathrm{~h}$ to deactivate and block the excess reactive groups remained on the graphene surface. Finally, the anti-CEA-modified GFETs were rinsed 
with deionized water and used for subsequent detection of target molecules.

\subsection{Detection of anti-CEA-modified GFET}

Anti-CEA-modified GFET operated at a source voltage of $0 \mathrm{~V}$, drain voltage of $0.1 \mathrm{~V}$ and top-gate voltage (Vgs) of $-0.3 \mathrm{~V}$ in phosphate buffer solution (PBS) at $\mathrm{pH}$ 7.2. In order to increase the Debye screening length, $1 \mu \mathrm{M}$ PBS was chosen as the gate electrolyte. The miniaturized $\mathrm{Ag} / \mathrm{AgCl}$ electrochemical reference electrode was chosen as the gate electrode. The concentration of CEA concentration was measured by the semiconductor parameter system (Keithley 4200).

\section{Results and Discussion}

\subsection{Device fabrication and modification}

GFETs were fabricated on silicon substrate with a $300 \mathrm{~nm}$ thick $\mathrm{SiO}_{2}$ layer. Using photolithography, metal deposition, and subsequent removal, titanium and gold were patterned on the substrate as source and drain electrodes. The single layer graphene film generated by chemical vapor deposition was transferred to metal electrodes. The channel of GFETs was defined by photolithography, and the surrounding graphene was etched by oxygen plasma under vacuum. The channel dimensions of these transistors were $25 \mu \mathrm{m}$ in width and $50 \mu \mathrm{m}$ in length. If no graphene layer on the Au electrodes, the absolute value of drain-source current was below 50 ÅA under different gate voltages shown in Fig. $\mathrm{S} 1$, and the drain voltage was $0.1 \mathrm{~V}$. The miniaturized $\mathrm{Ag} / \mathrm{AgCl}$ electrochemical reference electrode was immersed in the reactive chamber as electrolyte-gate of GFETs. The optical micrograph of the graphene channel on the substrate is shown in Fig. 1(a), and a schematic diagram of the solution-gated

GFET biosensor is shown in Fig. 1(b). If GFET had no extra encapsulation, the electrolyte-gated GFET 
couldn't be constructed and the graphene channel between drain and source worked as a resistance. The relationship curves between drain-source current and drain-source voltage were measured twice as shown in Fig. S2.

Anti-CEA modified GFET was constructed by modifying graphene. In order to minimize the effects of modification on the intrinsic properties of graphene, a non-covalent method was used to immobilize capture probes on the graphene surface. 1-pyrenebutanoic acid succinimidyl ester (PYR-NHS) served as a linker to conjugate Anti-CEA so that these modified GFETs could be used for detecting target cancer molecules. The schematic diagram depicting the modification steps of GFET is presented in Fig. 1(c). The interaction between the Au electrode and PYR-NHS was analyzed to reflect the resistance of Au electrode and was shown in Fig. S3(a), and no obvious resistance value changes of Au electrode indicated that Au electrode bound with PYR-NHS rarely as shown in Fig. S3(b).

\section{Insert Fig. 1}

The graphene channel of GFET was identified by Raman spectroscopy. Raman spectroscopy of bare graphene and PYR-NHS-modified graphene is shown in Fig. 2(a), revealing the non-covalent modification of PYR-NHS on the graphene surface. Two peaks, namely, a G band at $\sim 1590 \mathrm{~cm}^{-1}$ and a 2D band at $\sim 2687 \mathrm{~cm}^{-1}$, were observed in the typical Raman spectra of bare graphene and PYRNHS-modified graphene as shown in Fig. 2(a). The almost unchanged position of the 2D Lorentzian peak at $2687 \mathrm{~cm}^{-1}$ after PYR-NHS incubation revealed that the electric network of graphene remained unperturbed (Kodali et al. 2011). As shown in Fig. 2(a), there are small D peaks in Raman spectroscopy of bare graphene and PYR-NHS modified graphene, which indicated that some defects existed in the graphene channel and could bring in defects scattering. The intensity ratio (I (2D)/I (G)) between 2D band and G band decreased from 1.36 to 0.84 when PYR-NHS bound to graphene. These results 
indicated that Roman spectroscopy of PYR-NHS modified graphene was similar to that of bilayer graphene (Graf et al. 2007), which revealed that the binding of pyrenyl group, being highly aromatic in nature, on graphene, seemed to form bilayer graphene. All spectra are averaged from Raman mapping of 50 spots on graphene channel. This observation was verified by X-ray photoelectron spectroscopy (XPS) measurements. The C1s peaks of bare graphene and PYR-NHS-modified graphene are shown in Fig. 2(b). The results of XPS indicated that the C1s peak corresponding to $\mathrm{sp}^{2}$ hybridization remained unchanged after PYR modification as shown in Fig. 2(b). The conversion of carbon centers from $\mathrm{sp}^{2}$ to $\mathrm{sp}^{3}$ changed the C1s peak into a wide envelope (Bekyarova et al. 2009), which was not observed in our studies.

Similarly, the obvious shifts of transfer characteristic curves of GFETs were observed after the treatment of PYR-NHS as shown in Fig. 3(a). The evident shifts of transfer characteristic curves in Fig. 3(a) indicated the modification of PYR-NHS served as a doping effect on the graphene. These results were in accordance with the features of non-covalent modification.

\section{Insert Fig. 2}

\subsection{Characterization of Covalent Conjugation of Antibodies}

After modifying the anti-CEA on the graphene, the transfer and output characteristic curves of antiCEA-modified GFET were evaluated and were shown in Fig. 3(b) and Fig. 4(a) respectively. The transfer and output characteristics of anti-CEA-modified GFETs in Fig. 3(b) and Fig. 4(a) indicated that the anti-CEA modification process did not destroy the intrinsic properties of the graphene. From the results in Fig. 3(b), we could conclude that the difference between two neighbouring Ids in the Section A (red box) is much larger than that in the Section B (blue box). So the gate voltages in section A were better choices in our measurement. According to the driving drain-source voltage (Vds) 
suggested by other literatures (Huang et al. 2010; Ohno et al. 2010a), so we operated at a source voltage of $0 \mathrm{~V}$, drain voltage of $0.1 \mathrm{~V}$ and top-gate voltage $(\mathrm{Vgs})$ of $-0.3 \mathrm{~V}$ in our measurement. The mobility of anti-CEA-modified GFET was estimated to be $1400 \mathrm{~cm}^{2} /(V \cdot s)$ in the Supplementary Material. The leakage currents were measured under different top gate voltages while the drain voltage was $0 \mathrm{~V}$. Compared with the values of the net change in drain currents, the leakage currents of anti-CEAmodified GFET in Fig. 4(b) were below 100 nA, which could be considered negligible.

\section{Insert Fig. 3}

Atomic Force Microscopy (AFM) and Electrochemical Impedance Spectroscopy (EIS) were used to observe changes in the graphene channel before and after modification (detailed information regarding experimental results is shown in Supplementary Material). Upon addition of anti-CEA, the height in some parts of the graphene channel increased from $1 \mathrm{~nm}$ to about 7 10 nm, which is shown in Fig. S4. The graphene modified by PYR-NHS as well as the graphene modified by PYR-NHS bound to antiCEA demonstrated a gradual increase in interfacial charge transfer resistance of graphene as shown in Fig. S5.

X-ray photoelectron spectroscopy (XPS) was also used to confirm successful conjugation of anti-CEA on the graphene surface via the succinimidyl ester group. The recorded nitrogen (N1s) spectra of XPS for bare graphene, PYR-NHS modified graphene and anti-CEA-modified graphene by PYR-NHS are shown in Fig. 5(a). In Fig. 5(a), strong N1s peaks were observed after immobilization of anti-CEA onto graphene using the linker PYR-NHS. Controls demonstrated that a much weaker N1s peak was observed on the surface of PYR-NHS-modified graphene, and that the nonspecific binding of anti- 
CEA on the graphene surface was negligible. Hence, XPS results confirmed that PYR-NHS interacted with graphene non-covalently, and that the linker PYR-NHS could be used to immobilize the anti-CEA antibody on the graphene surface.

\section{Insert Fig. 4}

\subsection{Real-time Monitoring of CEA proteins}

Real-time monitoring of CEA proteins was performed using the semiconductor parameter system. All protein solutions were diluted by phosphate buffer solution (PBS). First, the specific sensing characteristics of anti-CEA-modified GFET were assessed. Fig. 5(b) shows the time dependence curve of the drain-source current (Ids) after adding the same value of control PBS, carcinoembryonic antigen (CEA), neuron specific enolase (NSE) and cytokeratin-19-fragment (CYFRA21-1). As shown in the specific detection curves of anti-CEA-modified GFET in Fig. 5(b), when PBS, CYFRA21-1 and NSE were added to the buffer solution, the drain current (Ids) showed no obvious increase. While introducing the target CEA protein, a large increase in drain current caused by the binding of CEA was observed. To accelerate the reaction between anti-CEA and CEA protein, the solution was stirred for several seconds after the addition of each protein. When the proteins were added in the channel of GFET, the drain current first decreased and then stabilized. This phenomenon is mainly due to the equilibrium time of the solution system after stirring. When the target protein was introduced into the graphene channel, the drain current of bare GFET was slightly decreased while that of the anti-CEA-modified GFET dramatically increased, as shown in Fig. 5(b).

These results demonstrate that the negatively charged CEA proteins were highly bound by the antiCEA-modified GFET, resulting in an increase in the drain current upon addition of CEA. Interestingly, 
the addition of control proteins NSE and CYFRA21-1 did not induce a similar increase in drain current, which indicates that any potential nonspecific binding of graphene with non-target proteins is likely negligible. Taken together, anti-CEA-modified GFET demonstrates high specificity in detection of CEA proteins.

Second, the drain-source current of anti-CEA-modified GFET was monitored at various CEA protein concentrations. The target CEA proteins at concentrations of $100 \mathrm{pg} / \mathrm{ml}, 1 \mathrm{ng} / \mathrm{ml}, 10 \mathrm{ng} / \mathrm{ml}, 50 \mathrm{ng} / \mathrm{ml}$ and $100 \mathrm{ng} / \mathrm{ml}$ were introduced into the channel of anti-CEA-modified GFET in Fig. 6(a) while the drain current was monitored in real-time. The isoelectric point of CEA protein was approximately 4.4 4.7 (Casey and Kofinas 2008), indicating that these target molecules were negatively charged in PBS. The mechanism of action for anti-CEA modified GFET is that CAE proteins adsorbed on the surface of the graphene act as electron donors, resulting in conductance changes. For this reason, the drain current increased stepwise after injection of the target CEA at each concentration, which is shown in Fig. 6(a). The output characteristic curves of anti-CEA-modified GFET with constant drain voltages (0.1V) under different CEA concentrations were also shown in Fig. S6, and the drain current increased with the concentration of CEA proteins. The net change in drain current $(\Delta \mathrm{I})$ plotted as a function of CEA concentration is shown in Fig. 6(b). The LOD of anti-CEA-modified GFETs was estimated to be less than $100 \mathrm{pg} / \mathrm{ml}$, which is much smaller than the cut-off value $(5 \mathrm{ng} / \mathrm{ml})$ in clinical diagnosis. As such, the high sensitivity of this biosensor demonstrates promising potential in clinical applications and point-of-care cancer diagnostics.

\section{Insert Fig. 5}




\subsection{Estimation of Dissociation Constant}

Lastly, using anti-CEA-modified GFETs, the dissociation constant $\left(\mathrm{K}_{\mathrm{d}}\right)$ for the interaction between anti-CEA and CEA protein was estimated by monitoring the drain current (Ids) at different CEA protein concentrations. The results revealed that the drain-source currents increased with an increasing CEA protein concentration. However, when the CEA concentrations increased to $100 \mathrm{ng} / \mathrm{ml}$, the drainsource currents began to saturate gradually as shown in Fig. 6(b). The quantity of net drain-source current $(\Delta \mathrm{I})$ was calculated as a function of CEA protein concentrations, as shown in Fig. 6(b). These data plotted to a nonlinear curve, indicating that the interaction between the CEA protein and AntiCEA on the Hill adsorption model previously described (Koopal et al. 1994; Ringot et al. 2007)

$$
\Delta \mathrm{I}=\Delta \mathrm{I}_{\max } C_{c e a}^{n} /\left(\mathrm{K}_{d}^{n}+C_{c e a}^{n}\right)
$$

Where $\mathrm{K}_{\mathrm{d}}$ is the dissociation constant of the interaction between CEA protein and anti-CEA antibody, $\Delta \mathrm{I}_{\max }$ is the saturated net drain-source current, $C_{c e a}$ is the protein concentration, $\mathrm{n}$ is Hill cooperativity coefficient of the binding interaction.

\section{Insert Fig. 6}

According to the fitted red curve shown in Fig. $6(\mathrm{~b}), \Delta \mathrm{I}_{\max }, \mathrm{K}_{\mathrm{d}}$ and $\mathrm{n}$ were estimated to be 3.535 $\mu \mathrm{A}, 11.442 \mathrm{ng} / \mathrm{ml}$ and 0.237 respectively. The correlation coefficient $\left(\mathrm{R}^{2}\right)$ was above 0.98 in this experimental results fitting, which would be used to determine the best-fitting isotherm to the experimental data. The calculated value of $\mathrm{n}$ is less than 1 , which indicated the negative cooperativity in binding between the CEA and Anti-CEA. The molecular weight of CEA protein is about $180 \mathrm{kD}$ (Haggarty et al. 1986). As such, this dissociation constant could also be converted into $6.35 \times 10^{-11} \mathrm{M}$. 
While the dissociation constant between the CEA protein and anti-CEA antibody has been investigated previously (Morris et al. 1975; Nap et al. 1992), and determined to vary from $4 \times 10^{-12} \mathrm{M}$ to $1 \times 10^{-7}$ M. Therefore, the value of the resulting dissociation constant determined in our study using anti-CEAmodified GFETs was in accordance with previously reported results, indicating a high affinity between CEA protein and anti-CEA. Thus, the present antibody-modified GFET not only shows a high sensitivity to specifically detect target molecules, but also could be an effective tool to estimate dissociation constants for capture probes and target molecules.

\section{Conclusions}

A label-free antibody-modified graphene immunosensor based on non-covalent modification was developed and characterized in this study. Anti-CEA was immobilized in a single-layer graphene channel using a PYR-NHS linker. Raman spectroscopy, X-ray photoelectron spectroscopy, AFM and EIS confirmed the successful modification of the graphene channel using a PYR-NHS linker bound to anti-CEA antibody. The anti-CEA-modified GFET could achieve a LOD of CEA less than $100 \mathrm{pg} / \mathrm{ml}$ in real-time, with minimal nonspecific binding of non-target proteins. The dissociation constant $\left(\mathrm{K}_{\mathrm{d}}\right)$ for the interaction between anti-CEA and CEA protein was estimated to be $6.35 \times 10^{-11} \mathrm{M}$ by hill model. These results indicated the high binding affinity of anti-CEA-modified GFET, and demonstrated the wide potential of this biosensor for clinical applications and point-of-care medical diagnostics.

\section{Acknowledgment}

This work was supported by Grants from the National Basic Research Program of China (973 Program)(No2012CB933303), the National Science Foundation of China (No. 61571429, 
61401442,61571428 and 61571077).

\section{Appendix A. Supplementary Material}

Electric detection of PYR-NHS and Anti-CEAs modified GFET, Atom Force Microscope (AFM) observation of GFET, Electrochemical Impedance Spectroscopy (EIS) Characterization of Graphene Modification.

\section{References}

Bekyarova, E., Itkis, M.E., Ramesh, P., Berger, C., Sprinkle, M., de Heer, W.A., Haddon, R.C., 2009. Chemical modification of epitaxial graphene: spontaneous grafting of aryl groups. J Am Chem Soc 131(4), 1336-1337.

Biomarkers Definitions Working, 2001. Biomarkers and surrogate endpoints: preferred definitions and conceptual framework. Clin Pharmacol Ther 69(3), 89-95.

Casey, B.J., Kofinas, P., 2008. Selective binding of carcinoembryonic antigen using imprinted polymeric hydrogels. J Biomed Mater Res A 87(2), 359-363.

Chen,R.J., Z.Y., Wang,D.W., Dai,H.J., 2001. Noncovalent Sidewall Functionalization of SingleWalled Carbon Nanotubes Immobilization for Protein Immobilization. J Am Chem Soc 123, 38383839 .

Chikkaveeraiah, B.V., Bhirde, A.A., Morgan, N.Y., Eden, H.S., Chen, X., 2012. Electrochemical immunosensors for detection of cancer protein biomarkers. ACS Nano 6(8), 6546-6561.

Graf,D.,Molitor,F., Ensslin,K., Stampfer,C., Jungen, A., Hierold,C., Wirtz,L.,2007. Spatially Resolved Raman Spectroscopy of Single- and Few-Layer Graphene. Nano Lett 7(2),238-242.

Geim, A.K., Novoselov, K.S., 2007. The rise of graphene. Nat Mater 6(3), 183-191.

Georgakilas, V., Otyepka, M., Bourlinos, A.B., Chandra, V., Kim, N., Kemp, K.C., Hobza, P., Zboril, R., Kim, K.S., 2012. Functionalization of graphene: covalent and non-covalent approaches, derivatives and applications. Chem Rev 112(11), 6156-6214.

Giljohann, D.A., Mirkin, C.A., 2009. Drivers of biodiagnostic development. Nature 462(7272), 461 464.

Goldsmith, S.J., 1975. Radioimmunoassay: review of basic principles. Semin Nucl Med 5, 125-152.

Haggarty, A., Legler, C., Krantz, M.J., Fuks, A., 1986. Epitopes of carcinoembryonic antigen defined by monoclonal antibodies prepared from mice immunized with purified carcinoembryonic antigen or HCT-8R cells. Cancer Res 46(1), 300-309.

Huang, Y., Dong, X., Shi, Y., Li, C.M., Li, L.J., Chen, P., 2010. Nanoelectronic biosensors based on CVD grown graphene. Nanoscale 2(8), 1485-1488.

Jaegfeldt, H., Kuwana,T.,Johanssont,G., 1983. Electrochemical Stability of Catechols with a Pyrene Side Chain Strongly Adsorbed on Graphite Electrodes for Catalytic Oxidation of 
Dihydronicotinamide Adenine Dinucleotide. J. Am. Chem. SOC 105, 1805-1814.

Katz, E., 1994. Application of bifunctional reagents for immobilization of proteins on a carbon electrode surface:oriented immobllization of photosynthetic reaction centers. Journal of EIectroanaJytical Chemistry 365, 157-164.

Kingsmore, S.F., 2006. Multiplexed protein measurement: technologies and applications of protein and antibody arrays. Nat Rev Drug Discov 5(4), 310-320.

Kodali, V.K., Scrimgeour, J., Kim, S., Hankinson, J.H., Carroll, K.M., de Heer, W.A., Berger, C., Curtis, J.E., 2011. Nonperturbative chemical modification of graphene for protein micropatterning. Langmuir 27(3), 863-865.

Koopal,L.K.,van Riemsdijk,W.H.,de Wit,J.C.M.,Benedetti,M.F., 1994. Analytical Isotherm Equations for Multicomponent Adsorption to Heterogeneous Surfaces. J Colloid Interf Sci 166, 51-60.

Morris, J.E., Egan, M.L., Todd, C.W., 1975. The binding of carcinoembryonic antigen by antibody and its fragments. Cancer Res 35(7), 1804-1808.

Nap, M., Hammarstrom, M.L., Bormer, O., Hammarstrom, S., Wagener, C., Handt, S., Schreyer, M., Mach, J.P., Buchegger, F., von Kleist, S., et al., 1992. Specificity and affinity of monoclonal antibodies against carcinoembryonic antigen. Cancer Res 52(8), 2329-2339.

Novoselov, K.S., Geim, A.K., Morozov, S.V., Jiang, D., Zhang, Y., Dubonos, S.V., Grigorieva, I.V., Firsov, A.A., 2004. Electric field effect in atomically thin carbon films. Science 306(5696), 666-669.

Ohno, Y., Maehashi, K., Matsumoto, K., 2010a. Chemical and biological sensing applications based on graphene field-effect transistors. Biosens Bioelectron 26(4), 1727-1730.

Ohno, Y., Maehashi, K., Matsumoto, K., 2010b. Label-free biosensors based on aptamer-modified graphene field-effect transistors. J Am Chem Soc 132(51), 18012-18013.

Ohno, Y., Maehashi, K., Yamashiro, Y., Matsumoto, K., 2009. Electrolyte-gated graphene field-effect transistors for detecting $\mathrm{pH}$ and protein adsorption. Nano Lett 9(9), 3318-3322.

Parviz, D., Das, S., Ahmed, H.S., Irin, F., Bhattacharia, S., Green, M.J., 2012. Dispersions of noncovalently functionalized graphene with minimal stabilizer. ACS Nano 6(10), 8857-8867.

Posthuma-Trumpie, G.A., Korf, J., van Amerongen, A., 2009. Lateral flow (immuno)assay: its strengths, weaknesses, opportunities and threats. A literature survey. Anal Bioanal Chem 393(2), 569582.

Ringot, D., Lerzy, B., Chaplain, K., Bonhoure, J.P., Auclair, E., Larondelle, Y., 2007. In vitro biosorption of ochratoxin A on the yeast industry by-products: comparison of isotherm models. Bioresour Technol 98(9), 1812-1821.

Schedin, F., Geim, A.K., Morozov, S.V., Hill, E.W., Blake, P., Katsnelson, M.I., Novoselov, K.S., 2007. Detection of individual gas molecules adsorbed on graphene. Nat Mater 6(9), 652-655.

Stern, E., Wagner, R., Sigworth, F.J., Breaker, R., Fahmy, T.M., Reed, M.A., 2007. Importance of the Debye screening length on nanowire field effect transistor sensors. Nano Lett 7(11), 3405-3409.

Sun, Z., Kohama, S.I., Zhang, Z., Lomeda, J.R., Tour, J.M., 2010. Soluble graphene through edgeselective functionalization. Nano Research 3(2), 117-125.

Voller, A., Bartlett, A., Bidwell, D.E., 1978. Enzyme immunoassays with special reference to ELISA techniques. J Clin Pathol 31(6), 507-520.

Xu, G., Abbott, J., Qin, L., Yeung, K.Y., Song, Y., Yoon, H., Kong, J., Ham, D., 2014. Electrophoretic and field-effect graphene for all-electrical DNA array technology. Nat Commun 5, 4866.

Zajac, A., Song, D., Qian, W., Zhukov, T., 2007. Protein microarrays and quantum dot probes for early cancer detection. Colloids Surf B Biointerfaces 58(2), 309-314. 


\section{Captions}

Fig. 1. (a) Optical micrograph of the graphene channel; (b) Schematic diagram of solution gated GFET biosensor; (c) The schematic diagram of all the modification steps for GFET.

Fig. 2. (a) Raman spectra (excitation: $532 \mathrm{~nm}$ ) of the graphene channel before and after PYR-NHS modification; (b) The C1s peaks of XPS spectra on the graphene surface before and after PYR-NHS modification.

Fig. 3. (a) Transfer characteristics of GFET before and after modification with PYR-NHS; (b) Transfer characteristic curves of anti-CEA-modified GFET under different drain voltages

Fig. 4. (a) Output characteristic curves of anti-CEA-modified GFET under different gate voltages; (b) The leakage drain currents of several Anti-CEA modified GFETs, the insert figure shows the details of curves.

Fig. 5. (a) The recorded nitrogen spectra of XPS for bare graphene, PYR-NHS modified graphene and Anti-CEA modified graphene by PYR-NHS; (b) The time dependence curve of the drain-source current when adding PBS, NSE, CYFRE21-1 and CEA

Fig. 6. (a) The time dependence curve of the drain-source current at various CEA concentration; (b) The net drain-source current at different CEA concentrations and a fitting red curve based on Hill 
adsorption model is presented.
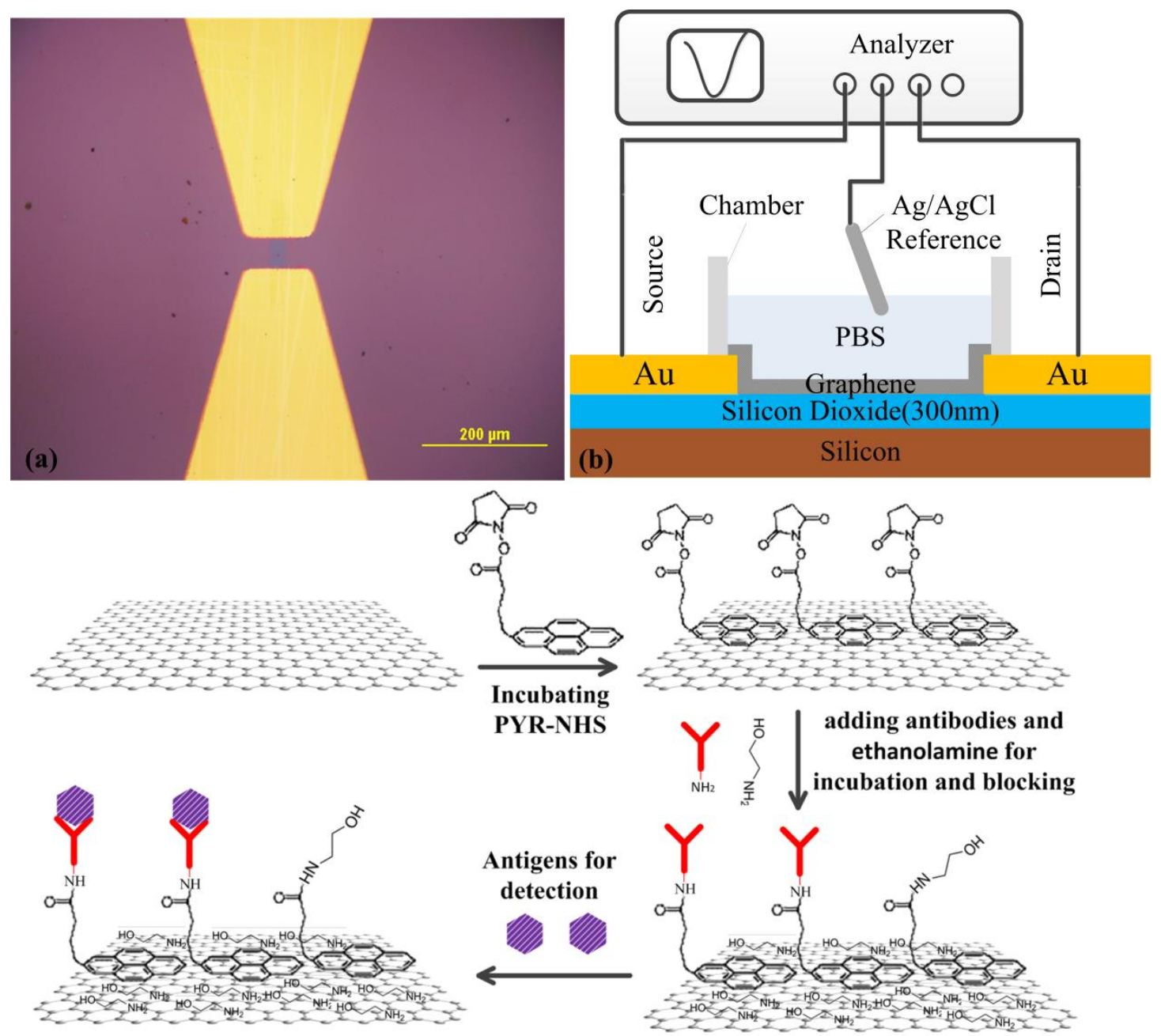

(c)
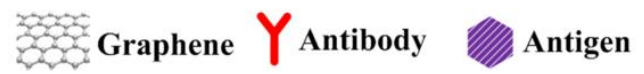

Fig. 1. (a) Optical micrograph of the graphene channel; (b) Schematic diagram of solution gated GFET biosensor; (c) The schematic diagram of all the modification steps for GFET. 

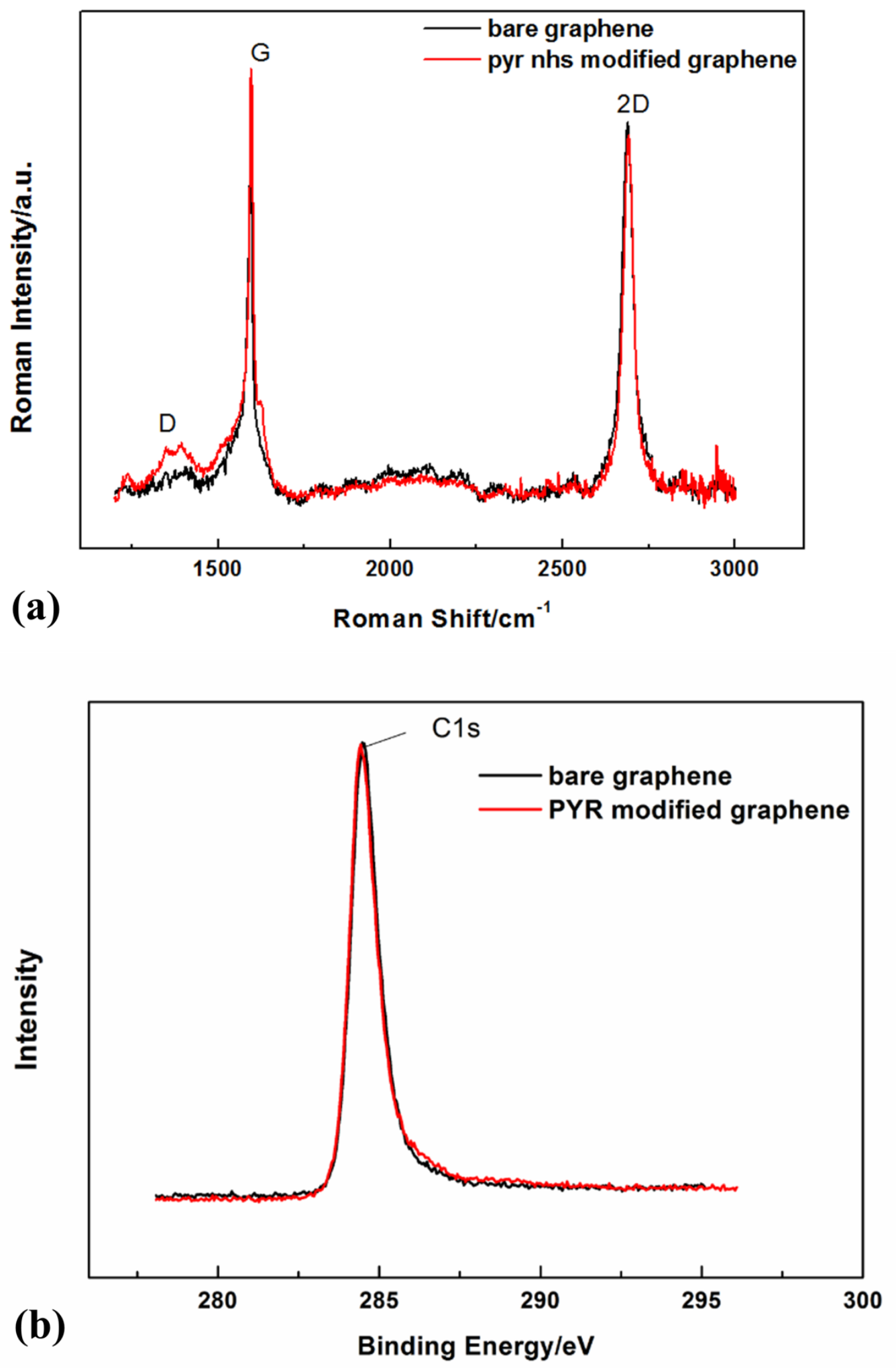

Fig. 2. (a) Raman spectra (excitation: $532 \mathrm{~nm}$ ) of the graphene channel before and after PYR-NHS modification; (b) The C1s peaks of XPS spectra on the graphene surface before and after PYR-NHS modification. 


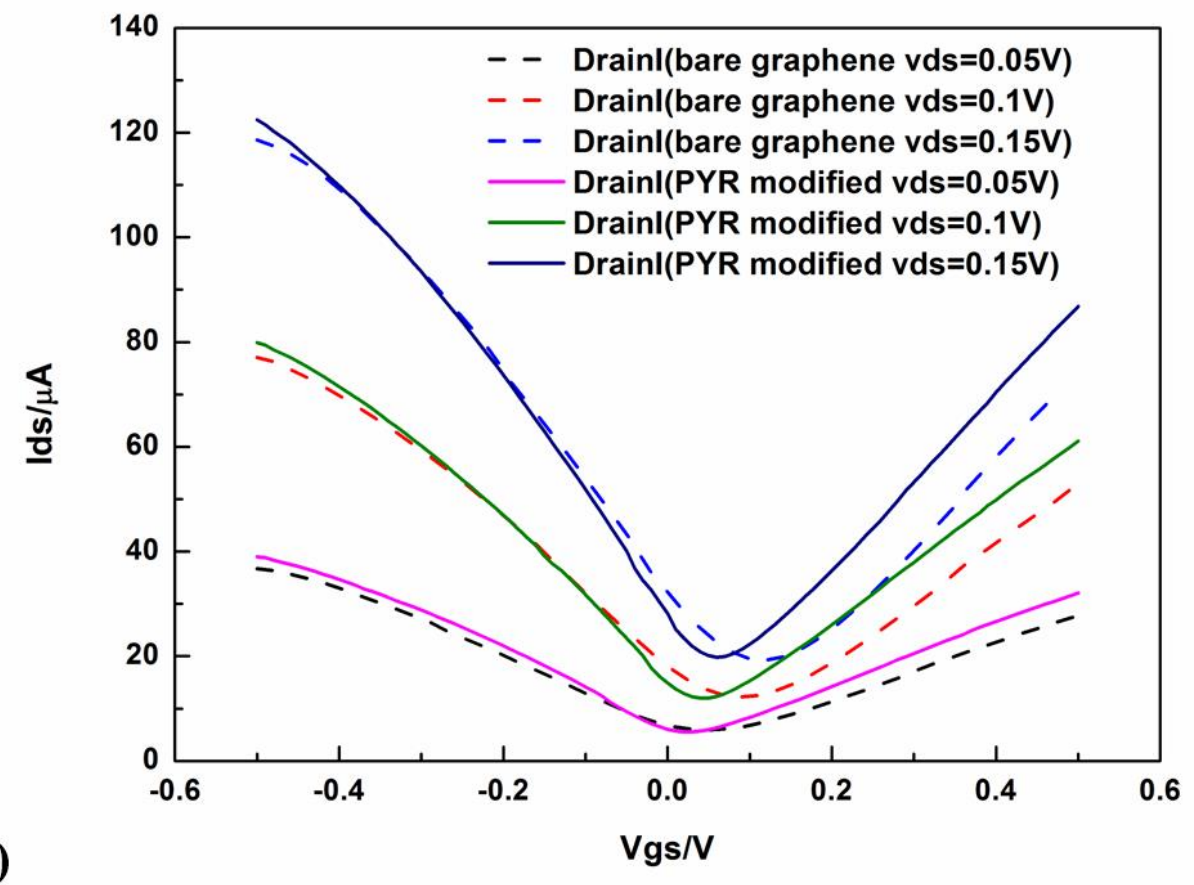

(a)

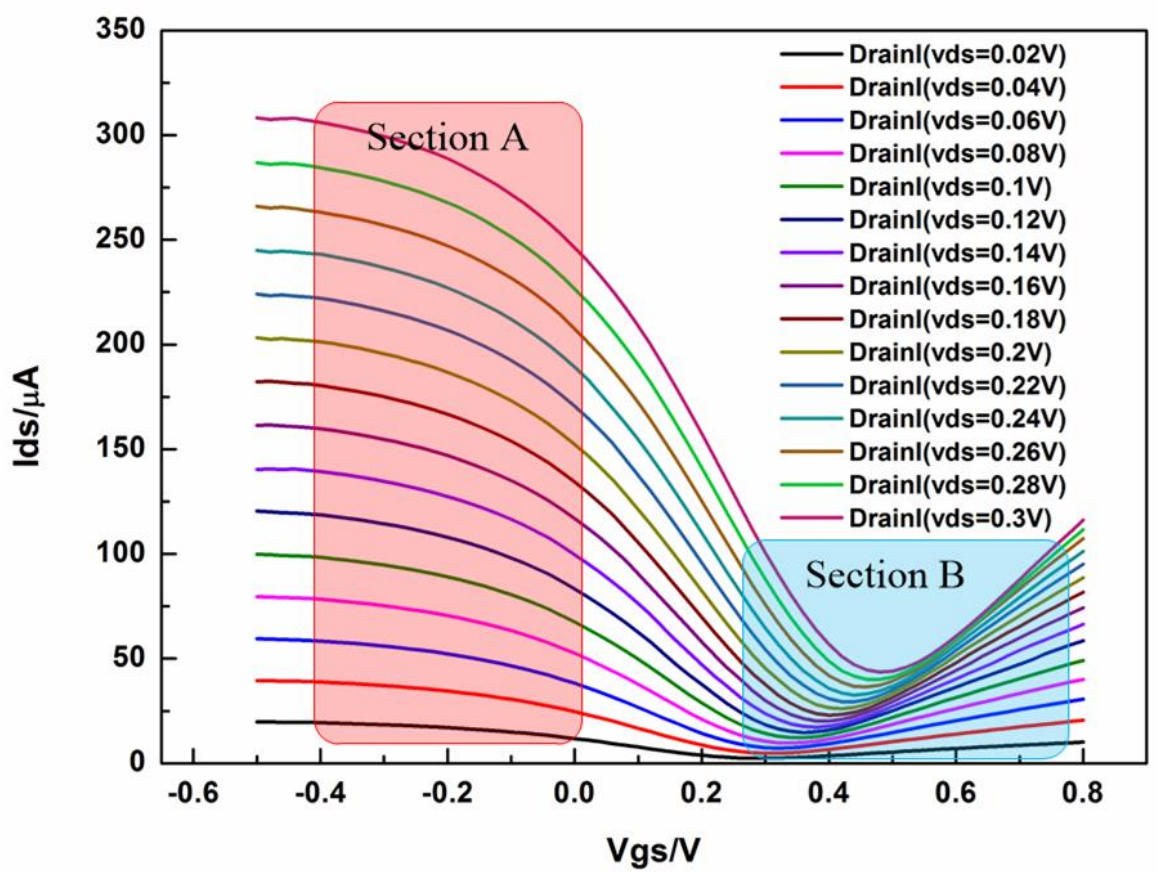

Fig. 3. (a) Transfer characteristics of GFET before and after modification with PYR-NHS; (b) Transfer characteristic curves of anti-CEA-modified GFET under different drain voltages 
(a)
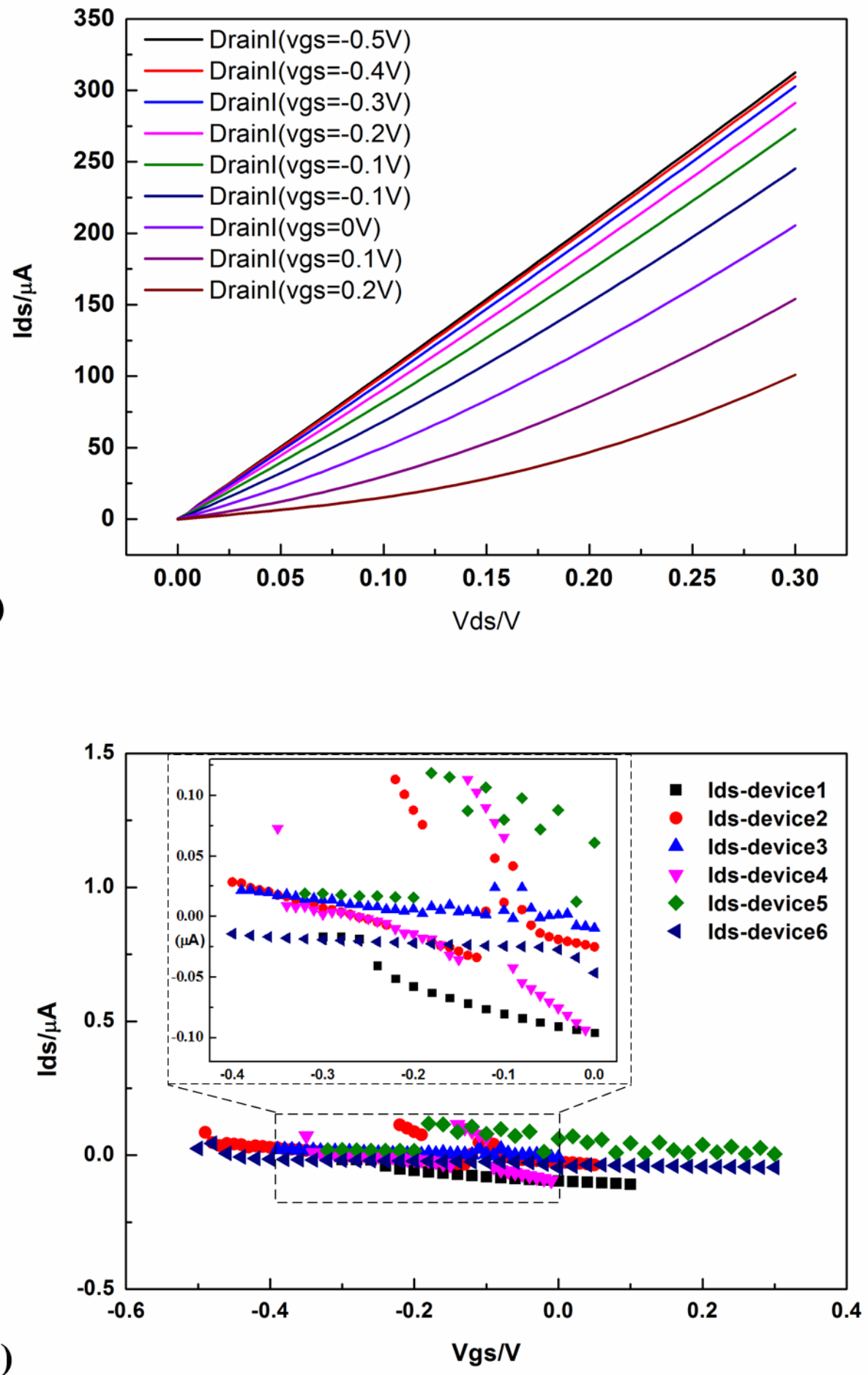

(b)

Fig. 4. (a) Output characteristic curves of anti-CEA-modified GFET under different gate voltages; (b)

The leakage drain currents of several Anti-CEA modified GFETs, the insert figure shows the details of curves. 


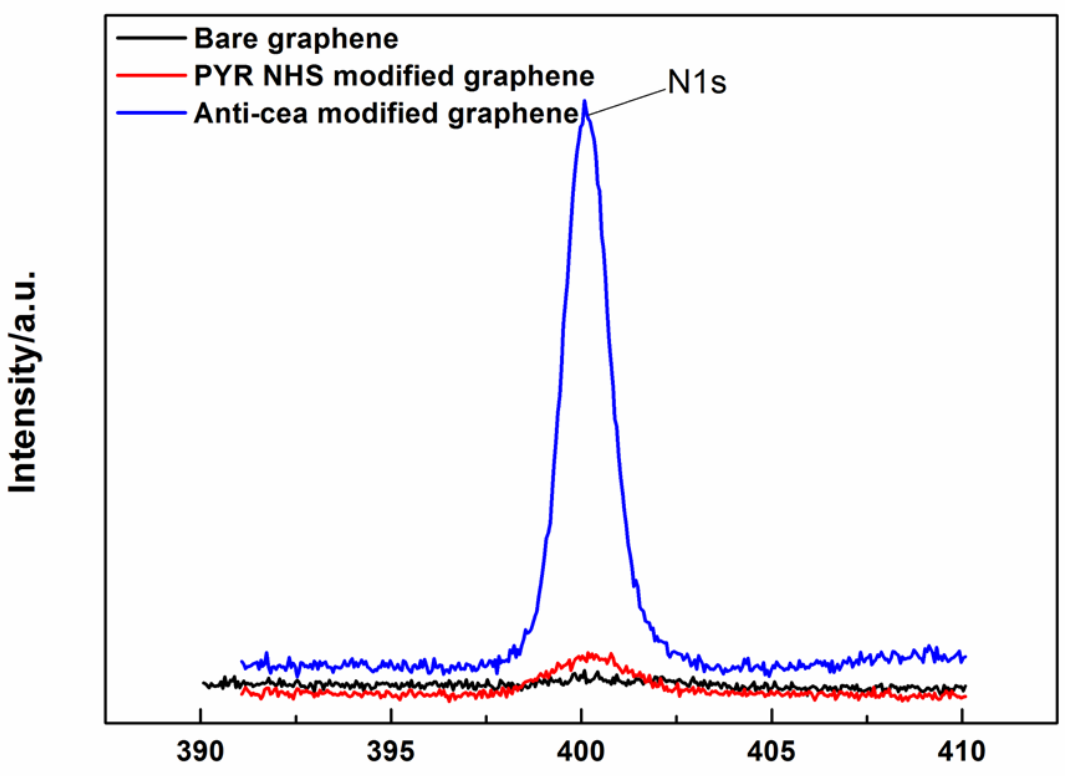

(a)

Binding Energy/eV

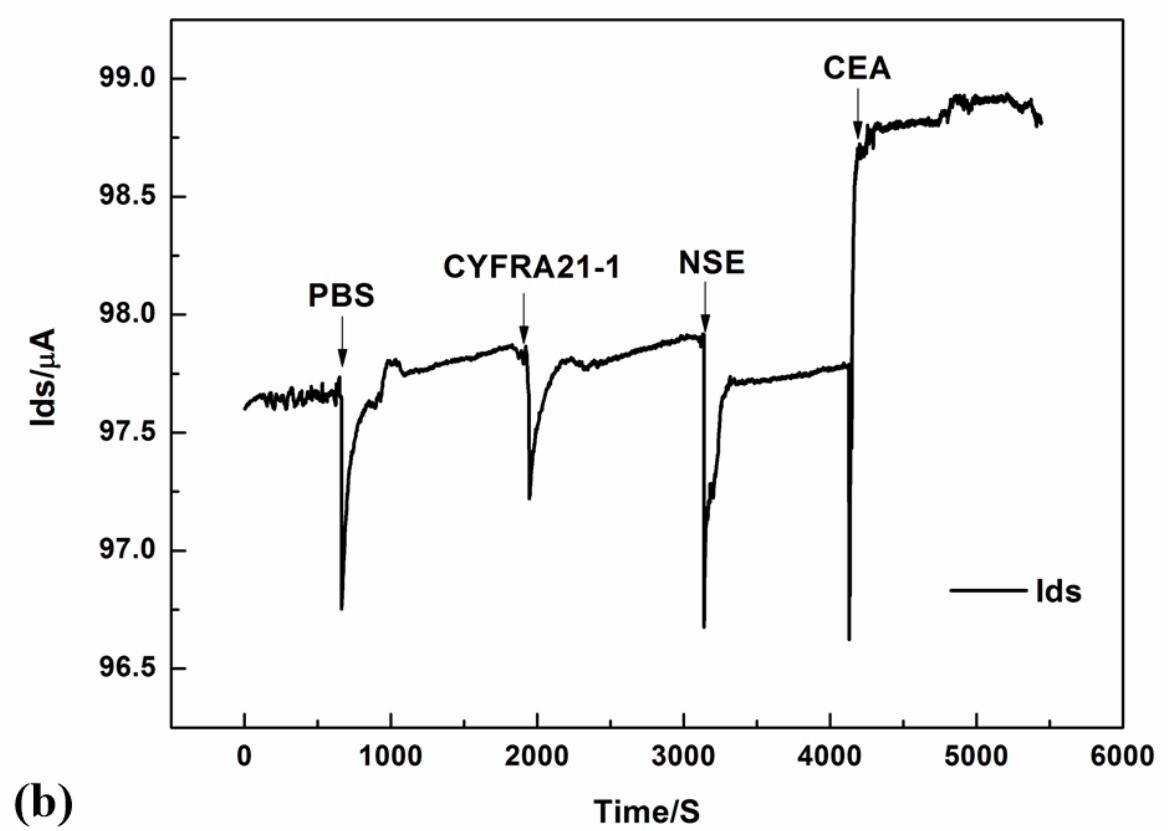

Fig. 5. (a) The recorded nitrogen spectra of XPS for bare graphene, PYR-NHS modified graphene and Anti-CEA modified graphene by PYR-NHS; (b) The time dependence curve of the drain-source current when adding PBS, NSE, CYFRE21-1 and CEA 


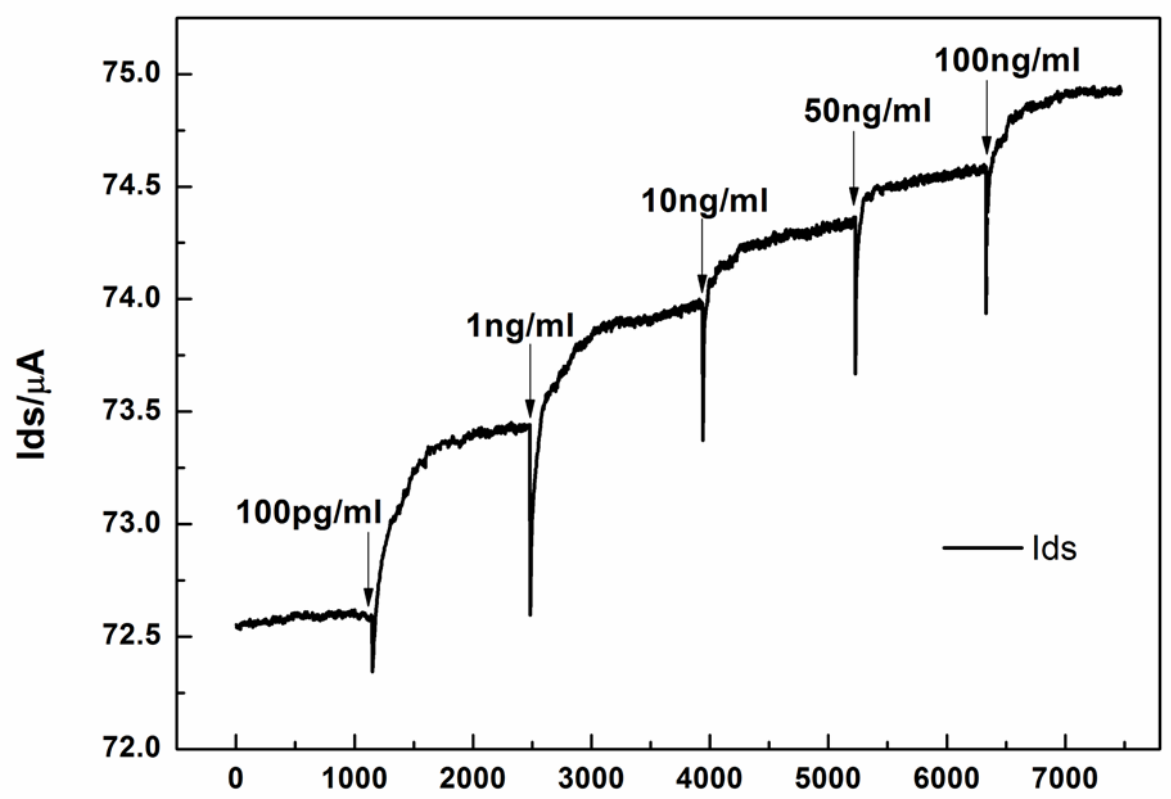

(a)

Time/s

(b)

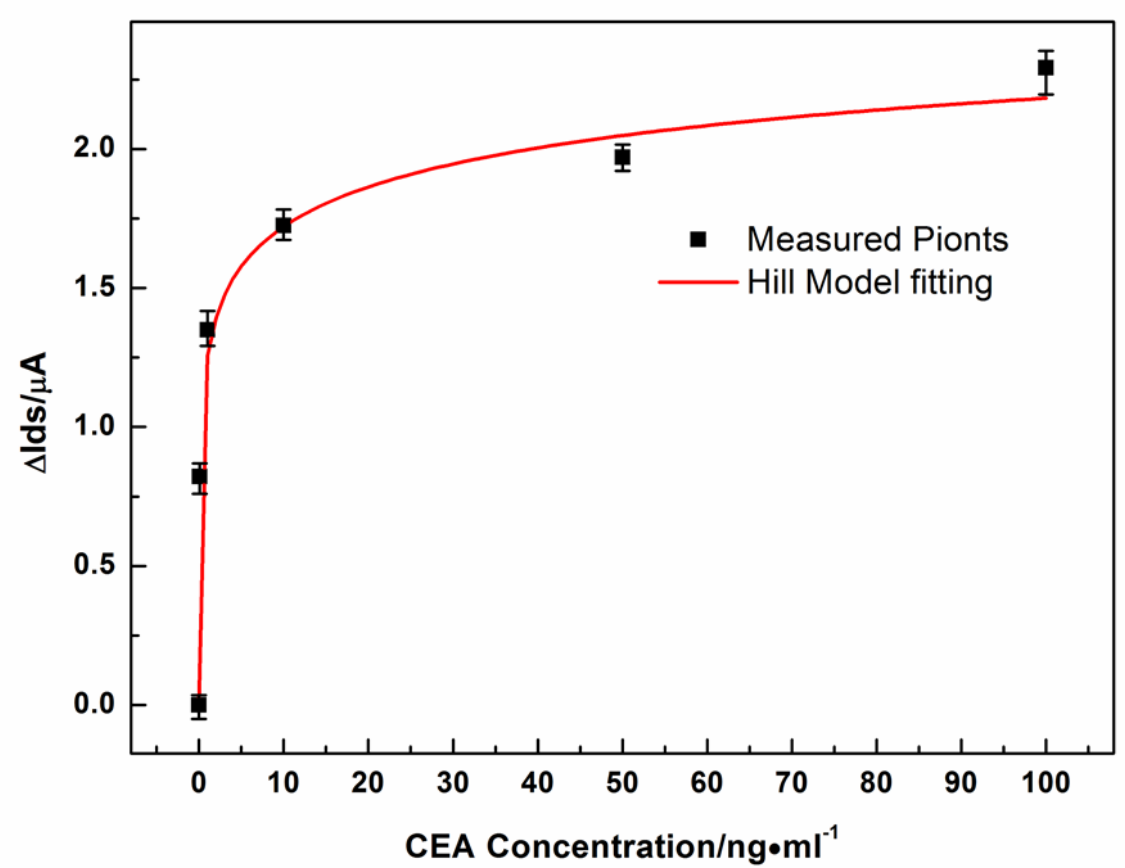

Fig. 6. (a) The time dependence curve of the drain-source current at various CEA concentration; (b)

The net drain-source current at different CEA concentrations and a fitting red curve based on Hill adsorption model is presented. 